\title{
EVALUACIÓN DE HABILIDADES DE CONSEJO BREVE EN EL ÁMBITO DE LAS ADICCIONES
}

\section{ASSESSMENT OF BRIEF COUNSELING SKILLS IN THE ADICTIONS SCENARIOS}

\author{
Silvia Morales Chainé ${ }^{1}$, K. Isela Martínez Martínez ${ }^{2}$, César Carrascoza Venegas ${ }^{3}$, A. Alelí Chaparro Caso López ${ }^{4}$ y M. José \\ Martínez Ruíz ${ }^{1}$ \\ ${ }^{1}$ Facultad de Psicología, Universidad Nacional Autónoma de México \\ ${ }^{2}$ Facultad de Psicología, Universidad Autónoma de Aguascalientes \\ ${ }^{3}$ Facultad de Estudios Superiores Iztacala, Universidad Nacional Autónoma de México \\ ${ }^{4}$ Universidad Autónoma de Baja California
}

\section{Abstract}

Drinking behavior and other use behaviors, in Mexico, has showed an increased tendency throughout the last years. We need to have some effective prevention strategies and brief counseling programs with easy access to the whole population. The goal of this study was to assess the brief counseling skills of the health and not health professionals (assess, dialog, goal establishment and companion) at the simulated interactions situations between a promoter and a factitious user throughout factorial design of 2 per 4: health professionals vs. non-health professionals at a) training with material, b) training without material, c) material without training, and d) without material and without training. Results show that the mean scores and score per scale were higher for the health professionals in the three training conditions. Both, training for skills acquisition and the possibility of having material of training, are important to brief counseling at the addictions prevention scenarios.

Keywords: brief counseling, addictions, health professionals.

\section{Resumen}

En las últimas dos décadas, el consumo de alcohol y otras drogas en México ha mostrado una tendencia al incremento. Es necesario disponer de estrategias en consejo breve para la prevención efectiva de las adicciones, de fácil acceso a la mayor parte de la población. El objetivo del presente trabajo fue evaluar las habilidades de consejo breve de los profesionales y no profesionales de la salud (Identifica, Dialoga, Establece metas y Acompaña ) en situaciones simuladas de interacción promotor usuario a través de un diseño factorial de 2 por 4: profesionales de la salud versus no profesionales de la salud en una de cuatro condiciones: a) entrenamiento con material, b) entrenamiento sin material, c) con material sin entrenamiento, y d) $\sin$ entrenamiento y sin material. Los resultados mostraron que la puntuación promedio y por escala, fue mayor para los profesionales de la salud en cualquiera de las tres condiciones de entrenamiento. Tanto el entrenamiento en habilidades como la disponibilidad del material son necesarios para llevar a cabo el consejo breve en la prevención de las adicciones.

Palabras clave: consejo breve, adicciones, profesionales de la salud.

$\begin{array}{ll}\text { Correspondencia: } & \text { Silvia Morales Chainé } \\ & \text { Facultad de Psicología } \\ & \text { Universidad Nacional Autónoma de México } \\ & \text { E-mail: smchaine@gmail.com }\end{array}$


Aun cuando, de acuerdo a Oficina de la ONU contra las Drogas y la Delincuencia (UNODC, por sus siglas en inglés, 2010) el consumo de drogas en el mundo se ha estancado, 200 mil personas al año mueren debido a éstas y una de cada 200 personas es dependiente. Uno de los grandes problemas con respecto al tratamiento de personas con adicciones consiste en la poca disponibilidad de este servicio. De acuerdo a la misma fuente, menos de cinco personas que necesitan ayuda la recibe. Como referencia nótese que durante 2010, 230 millones de personas ( $5 \%$ de la población mundial) tomaron drogas al menos una vez durante ese año, mientras que 27 millones desarrollaron el trastorno por consumo de drogas. A finales de siglo el número de usuarios dependientes se podría elevar a 300 millones. Anualmente, por consumo de alcohol mueren 2.3 millones y por consumo del tabaco 5.1 millones de personas.

En México, el consumo de drogas está aumentando principalmente entre los adolescentes. Por ejemplo, en el 2002 el .5\% de la población adolescente consumía marihuana, mientras que en el 2008 lo hizo el $1.2 \%$ y en 2011 el $1.3 \%$ de los jóvenes. Sin embargo, los estudiantes de secundaria y preparatoria consumen principalmente drogas legales, tales como el alcohol (42.9\%) y el tabaco (12.3\%). Respecto a las drogas ilegales, la marihuana es la más popular (1.3\%), seguida por la cocaína $(0.4 \%)$, de acuerdo a los datos proporcionados por la ENA (2011).

De acuerdo con Camacho (2012), un adolescente que comienza a ingerir alcohol, a fumar tabaco o a inhalar o aspirar sustancias tiene hasta 13 veces más probabilidades de usar posteriormente drogas como la heroína, la cocaína y las drogas de diseño. El alcohol ha sido la droga de inicio en el 38\% de los adultos adictos y el tabaco en el $40 \%$ de esta población. Aun cuando en Distrito Federal el $15.4 \%$ de los estudiantes de secundaria ha tenido contacto con las drogas, se considera que todavía no enfrenta problemas de adicción. No obstante, de acuerdo con la ENA (2011) en el último año, medio millón de ciudadanos en México aceptaron haber consumido alguna droga, como marihuana, cocaína e inhalables. Existen 2.4 consumidores de marihuana por cada uno de cocaína y, aunque las metanfetaminas e inhalables tienen una prevalencia baja entre los adolescentes, se consumen en la misma proporción que la cocaína. De acuerdo a la ENA de 2011, se sabe que los jóvenes, sobre todo los que no estudian o trabajan, son los más expuestos a las drogas debido a su baja percepción de riesgo y a los procesos de pertenencia e identificación social.

Con sustento en el Programa Nacional de Salud 2007-2012 y a partir de los daños ocasionados por el consumo de drogas en la vida individual, en las relaciones familiares y en los aspectos sociales, el Gobierno Federal promovió el entrenamiento de promotores de la salud en el consejo breve en adicciones (Tavera y Martínez, 2008). El entrenamiento se concretó mediante el Modelo Integral de Atención que consta de tres dimensiones: a) prevención y promoción, incluidos los niveles de prevención universal (dirigida a poblaciones con o sin riesgo y cuya finalidad consiste en evitar el consumo o retrasar la edad de inicio), selectiva (dirigida a grupos de riesgo, como aquellos de consumo experimental, deteniendo el avance a consumo problemático) e indicada (dirigida a evitar que avance el consumo y disminuir los problemas asociados al mismo); b) tratamiento, que comprende tanto la identificación temprana como el consejo e intervención breve (cognitivo conductual) oportuna, así como la intervención relacionada al uso regular de drogas, la tolerancia a las mismas y la búsqueda de actividades relacionadas con su obtención y consumo; y c) la rehabilitación, dirigida a quienes ya consumen drogas, con la intención de evitar que avance, así como disminuir los problemas asociados (consiste en el tratamiento ambulatorio o institucional y el tratamiento continuo, la reinserción social y la reducción del daño).

El consejo breve (Talavera y Martínez, 2008) está dirigido a la detección temprana de individuos que presentan abuso de sustancias con la finalidad de reducir el avance del problema. Es un tipo de intervención breve que permite una rápida evaluación e implementación de estrategias que favorecen el cambio de conducta (Bien, Miller y Tonigan, 1993; Cuijpers, Riper y Lemmers, 2004; Medina-Mora, 2005; Liska, Miller, Nemith, Jenkins, Nicktertert, Wesell y Ornstein, 2008). Es limitado en el tiempo de duración e incluye una evaluación inicial, asesoramiento breve y evaluación final. Por ejemplo, Winters, Fahnhorst, Botzet, Lee y Lalone, (2012) trabajaron con 315 adolescentes y sus familias a través de un ensayo clínico aleatorizado para evaluar el consejo breve. Los dividieron en tres grupos: el consejo solo para los adolescentes, el consejo para los adolescentes y sus padres y un grupo control con solo evaluación. Sus resultados indicaron que los adolescentes redujeron significativamente las conductas de consumo de drogas en las dos condiciones de consejo breve. Las ventajas del consejo breve consisten en que es de bajo costo, de rápida aplicación y de efectos inmediatos. Sus resultados son perdurables en el tiempo, puede llevarse a cabo en situaciones que van desde la atención de emergencia in situ, hasta su implementación en instituciones de salud pública o privada (Carrascosa, 2007).

El consejo breve consta de cinco pasos: Indaga (identificación, mediante cuestionarios del nivel de abuso o dependencia a alguna o varias drogas), Dialoga (informa a la persona sobre su nivel de consumo y las consecuencias que ha tenido y puede tener debidas al consumo). Establece metas (ayudar a la persona a establecer una meta de reducción o abstinencia para evitar las consecuencias negativas a corto y a largo plazo), Acompaña en el proceso de cambio (indagar el nivel de satisfacción de la persona con la asesoría de consejo breve, ya que una satisfacción alta puede propiciar que la persona continúe el proceso de consejo, mientras que una baja satisfacción puede indicar que la persona no regresará), y Seguimiento (detectar si ha habido cambios significativos en el 
consumo; si no han ocurrido se debe canalizar a la persona a otros tipos de tratamiento).

De este modo, IDEAS es una guía para los promotores que proporciona la información necesaria para prevenir que algún miembro de la familia tenga contacto con las drogas, o si ya es consumidor, proporcionar algún tratamiento de rehabilitación (Talavera y Martínez, 2008). En México, Martínez et al. (2008) evaluaron el efecto del consejo breve sobre el consumo de alcohol con 40 adolescentes en dos grupos aleatorizados: experimental y lista de espera. Los resultados indicaron que el consejo breve fue efectivo al reducir el patrón de consumo de alcohol entre los adolescentes del grupo experimental, observando diferencias significativas contra el grupo control en la fase de seguimiento a los tres y seis meses.

A pesar de la efectividad de los procedimientos de consejo breve, existen pocos esfuerzos en evaluar las habilidades profesionales o no profesionales en el campo del análisis conductual aplicado para la consejería breve. Por ejemplo, Whang, Fletcher y Fawcett, (1982) evaluaron el efecto de 40 horas de entrenamiento a 2 no profesionales de bajos recursos de un centro de servicio comunitario como consejeros y en solución de problemas a través de instrucciones escritas, práctica y retroalimentación de su ejecución por medio de un diseño de línea base múltiple entre participantes. Aunque, el entrenamiento no ocurrió en el ámbito de la prevención de las adicciones, es importante señalar que ellos encontraron que los procedimientos de entrenamiento fueron efectivos para la adquisición de habilidades de consejo y solución de problemas en los participantes no profesionales dentro la comunidad.

Morales y Vázquez (2011) han argumentado que los profesionales de la salud cuentan con el conocimiento teórico y las habilidades prácticas en el manejo del comportamiento para transmitirlas y manejarlas en situaciones reales a través del consejo y la intervención breve. Adicionalmente, Hughes y MacNaughton, (2002) señalan que proceso de entrenamiento no se debe tomar como una situación en la cual el profesional evalúa e impone la forma en que un usuario debe actuar, sino que en este proceso, el usuario o participante decide con base en sus expectativas y necesidades, las metas a lograr con la intervención (Baer, Wolf y Risley, 1968). Por lo que Morales y Vazquez (2011) llevaron a cabo un estudio con 294 profesionales de diversos estados del país para evaluar sus conocimientos en una intervención breve, a través de un diseño pre-experimental pre-post, utilizando un instrumento de 13 situaciones hipotéticas relacionadas con habilidades basadas en principios conductuales para resolver situaciones problemáticas en el ámbito de la crianza infantil (prevención universal en adicciones). El entrenamiento consistió en la presentación de contenidos, ensayos conductuales, modelamiento y retroalimentación. Los resultados mostraron que hubo un incremento significativo en los conocimientos sobre las habilidades en los profesionales de la salud, después del entrenamiento.
En el modelo de atención integral, la intervención y detección temprana se llevan a cabo mediante el consejo individual o familiar, intervenciones breves, intervenciones familiares, grupos de autoayuda, prevención de recaídas y cuidados posteriores, cuyo entrenamiento en profesionales requiere de evaluación. Para propósitos de este trabajo nos centraremos principalmente en el consejo breve, cuya finalidad consiste en prevenir la ocurrencia del contacto inicial con las drogas o, si el consumo ya está ocurriendo, ofrecer alternativas de intervención mediante la aplicación de una serie de técnicas agrupadas en el acrónimo IDEAS (Martínez, 2003; Martínez, Pedroza, Vacío, Jiménez y Salazar, 2008; Talavera y Martínez, 2008; Martínez, Salazar, Ruiz, Barrientos y Ayala, 2009) en los Centros Especializados para la atención de las adicciones.

El propósito del entrenamiento fue habilitar a profesionales y no profesionales de la salud de la salud en el consejo breve, que consiste en la prevención, identificación, canalización y en su caso para el tratamiento y el control de las adicciones, con la finalidad de mejorar la calidad de vida individual, familiar y social, dirigida sobre todo a niños y jóvenes, y evitar, de esta forma, el desarrollo de dependencia o algún otro tipo de daño o riesgo personal, familiar o social.

A partir de la escasa literatura en México sobre la evaluación de las habilidades durante el consejo breve y dada la importancia de la implementación óptima del programa para el logro de sus metas, el objetivo de este trabajo consistió en la evaluación de las habilidades de consejo breve en adicciones de los profesionales y no profesionales de la salud en el programa IDEAS a través de la evaluación de las habilidades prácticas adquiridas mediante situaciones simuladas relacionadas con la interacción promotor usuario y la aplicación de los pasos del programa.

\section{MÉTODO}

\section{Participantes}

Se evaluó a 412 personas de diferentes entidades e instituciones de la república mexicana invitados a ser promotores, por lo que los grupos de la investigación no pudieron ser homogéneos en cantidad o en nivel académico, ya que podrían ser profesionales de la salud (psicólogos, médicos, pedagogos, enfermeras) o de alguna otra profesión o actividad (maestros, abogados, voluntarios, amas de casa, etc.).

Los participantes se dividieron en dos muestras: 1) profesionales de la salud (133 personas), y 2) no profesionales de la salud (279 personas). El $77 \%$ de la población fueron mujeres, y el promedio de todos los participantes fue de 32 años de edad. En cuanto al nivel educativo, el $7 \%$ de los participantes estudiaron la primaria, el 10\% secundaria, el 23\% preparatoria, el $47 \%$ eran profesionales, el $10 \%$ una carrera 
trunca y el 3\% estudiaron un posgrado. El perfil profesional en

función del tipo de promotor se observa en la Tabla 1.

Tabla 1. Representa los promedios de edad y los porcentajes de sexo y escolaridad de los dos grupos de participantes: profesionales de la salud y no profesionales de la salud.

\begin{tabular}{|c|c|c|c|c|c|c|c|c|c|}
\hline & \multirow{2}{*}{$\begin{array}{c}\text { Edad } \\
\text { Promedio }\end{array}$} & \multicolumn{2}{|c|}{ Sexo } & \multicolumn{6}{|c|}{ Escolaridad } \\
\hline & & Femenino & Masculino & Primaria & Secundaria & Preparatoria & Profesional & $\begin{array}{l}\text { Carrera } \\
\text { trunca }\end{array}$ & Posgrado \\
\hline $\begin{array}{c}\text { Profesionales } \\
\text { de la salud }\end{array}$ & 32 & $75 \%$ & $25 \%$ & $0 \%$ & $1 \%$ & $6 \%$ & $85 \%$ & $4 \%$ & $4 \%$ \\
\hline $\begin{array}{c}\text { No } \\
\text { profesionales }\end{array}$ & 32 & $77 \%$ & $23 \%$ & $10 \%$ & $14 \%$ & $31 \%$ & $30 \%$ & $13 \%$ & $2 \%$ \\
\hline
\end{tabular}

\section{Instrumentos}

Se utilizó una Lista Cotejable de 28 habilidades del profesional, a través de un sistema de observación directa (Apéndice 1). La lista tiene tres posibilidades de calificación: no muestra la habilidad, en proceso y la domina. Se obtuvo un $85 \%$ de fiabilidad interjueces para la puntuación de la lista cotejable, un alpha de cronbach de .90 y una varianza explicada de $75 \%$. El análisis factorial por escalas demostró una medición valida de las mismas (ver tabla 2).

Tabla 2. Muestra la validez de las Escalas de la Lista Cotejable del Consejo Breve: Identifica, Dialoga, Establece metas y Acompaña.

Varianza explicada

\begin{tabular}{cc}
\hline Indaga & $65.76 \%$ \\
Dialoga & $51 \%$ \\
Establece & $61.81 \%$ \\
\hline Acompaña & $62.56 \%$ \\
\hline
\end{tabular}

\section{Procedimiento}

Se invitó a los participantes de diferentes Estados de la República Mexicana, a recibir el entrenamiento y/o material en consejo breve en el ámbito de las adicciones. Los participantes recibieron un entrenamiento teórico-práctico de cuatro horas impartido por 3 especialistas en el área de la investigación y tratamiento de las adicciones. Estos voluntarios fueron divididos en dos muestras: Profesionales de la Salud y no Profesionales que pasaron por una de cuatro condiciones: entrenamiento con material, entrenamiento sin material, con material sin entrenamiento, y sin entrenamiento y sin material. Finalmente fueron evaluados a través de una situación simulada por 17 evaluadores.

En la primera condición, los participantes recibieron el entrenamiento con material que consistió en cuatro horas donde se expuso la información y se llevaron a cabo el modelamiento, ensayos conductuales y la retroalimentación de las habilidades de cada fase del consejo. El material consistió en un cuadernillo de 18 hojas, dividido en los siguientes apartados: prologo, bienvenida, enfoques de prevención, intervención acorde al nivel de consumo, consejo breve, paso 1 indaga, paso 2 dialoga, paso 3 establecimiento de metas y planes de acción, paso 4 acompaña en el proceso de cambio, paso 5 seguimiento, tips para identificar si alguien está utilizando drogas y el papel de la familia del consumidor.

En la segunda condición, los participantes recibieron solo el entrenamiento de cuatro horas con la exposición de la información, el modelamiento, los ensayos conductuales y la retroalimentación. En la tercera condición, los participantes recibieron el material que consistió en la entrega del cuadernillo de 18 hojas. En la cuarta condición los participantes no recibieron ni material ni entrenamiento, permaneciendo en una lista de espera.

Para la situación simulada de evaluación, se citó a los participantes a una sesión individual de 30 minutos. La sesión consistió en el juego de roles de una evaluadora que realizaba el papel de usuaria de alcohol, a quien el participante debía atender. Las instrucciones al participante fueron:

"Lo que haremos a continuación es una situación simulada. Vamos a simular que usted atenderá a una mujer que viene a pedirle ayuda porque tiene problemas con su consumo de alcohol. Usted seguirá el proceso de atención habitual desde el saludo hasta la despedida del usuario, tratando de establecer el consejo breve con la usuaria. Le pido que si en algún momento se presenta una circunstancia en la que usted no esté familiarizado, no trate de terminar la situación e intente abordarla como lo haría en situación real. Yo voy adoptar el papel de esta mujer por lo que el tiempo que dure la situación mi comportamiento se apegará al de este personaje respondiendo a sus peticiones solo como usuaria." ¿Tiene alguna duda hasta aquí? Vamos a comenzar. 
Durante la situación simulada las evaluadoras, en su papel de usuarias, presentaron diez estímulos con base en un guión, que aprendieron de memoria. Mostraron 2 tipos de conductas, descritas por Miller y Rollnick (1999), características de la etapa de contemplación del usuario, en el mismo orden: Dos conductas verbales sin resistencia ("Tengo un problema con el alcohol, ya que cuando bebo me emborracho"), dos conductas de confrontación ("¿usted en verdad tiene experiencia en esto?"), dos de desesperanza ("no creo que pueda con esto, soy un desastre y no puedo cambiar"), dos conductas de defensa de sí mismo ("es normal que todos tomen para sentirse tranquilos y felices") y dos auto-descalificación ("Si no bebo no puedo divertirme ni socializar con la gente"). Terminada la situación la evaluadora agradecía la participación del usuario, daba retroalimentación sobre el apego de sus habilidades al consejo breve y se despedía del usuario.

Para el análisis de los datos se llevó a cabo un ANOVA de 4 (Condición de entrenamiento: con entrenamiento y material, con entrenamiento, con material, sin entrenamiento ni material) X 2 (Tipo de participante: profesional y no profesional). El análisis evaluó los efectos principales de la condición de entrenamiento y el tipo de participante sobre el nivel de habilidades en consejo breve, así como efectos simples de interacción entre el tipo de participante y la condición.

\section{RESULTADOS}

A partir de la evaluación de las habilidades generales adquiridas por los integrantes de cada muestra, los promedios de calificación fueron mayores para los profesionales de la salud en cualquiera de las tres primeras condiciones: cuando recibieron entrenamiento y el material $(M=9.5)$, cuando recibieron entrenamiento pero no contaron con material impreso $(M=9.65)$ y cuando solo recibieron el material $(M=9.5)$ que cuando no recibieron ninguno de los dos ( $M=6.38$ ), en comparación con los promotores que no eran profesionales de la salud: entrenamiento con material $(\mathrm{M}=7.86)$, entrenamiento $(M=7.07)$, solo con material $(M=5.82)$ y $\sin$ material ni entrenamiento $(M=5.8)$.

El análisis de varianza demostró un efecto de interacción del tipo de participante por condición de entrenamiento sobre las habilidades $(F(7,410)=14.96, p=.005)$. Aun cuando puede observarse un descenso en las puntuaciones de los profesionales conforme se modifica la condición de entrenamiento, este descenso no se acerca lo suficiente al promedio de los no profesionales, cuando ambos no reciben material ni entrenamiento (ver Figura 1).

En la tabla 3, se observan las puntuaciones promedio y las desviaciones estándar obtenidas por los participantes durante las fases: Indaga, Dialoga, Establece metas y Acompaña. Los promedios de los profesionales de la salud para las primeras tres condiciones de entrenamiento fueron mayores que los promedios de los promotores que no son profesionales de la salud, en todas las fases: indaga, dialoga, establece metas y acompaña. No obstante, las puntuaciones de los profesionales de la salud que no reciben entrenamiento ni material son similares a los de los no profesionales en las fases indaga y dialoga y se mantienen elevadas en Establece metas y acompaña.

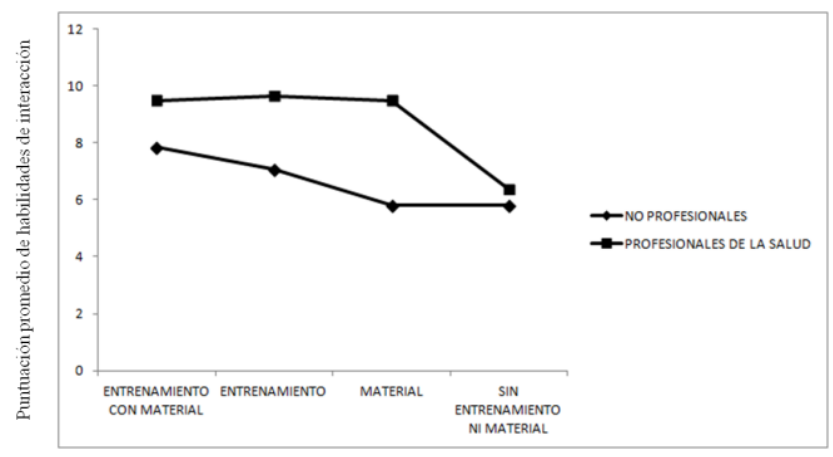

Figura 1. Efectos de interacción entre el promotor de la salud y la condición de entrenamiento sobre las habilidades generales de consejo breve.

Tabla 3. Representa los promedios y desviaciones estándar de los profesionales de la salud versus no profesionales en las cuatro condiciones de entrenamiento, para las 4 fases del consejo breve.

\begin{tabular}{|c|c|c|c|c|c|c|c|c|c|}
\hline & & \multicolumn{2}{|c|}{ Indaga } & \multicolumn{2}{|c|}{ Dialoga } & \multicolumn{2}{|c|}{ Establece } & \multicolumn{2}{|c|}{ Acompaña } \\
\hline & & $\bar{X}$ & D.E. & $\bar{X}$ & D.E. & $\bar{X}$ & D.E. & $\bar{X}$ & D.E. \\
\hline \multirow{2}{*}{$\begin{array}{c}\text { Entrena } \\
\text { miento } \\
\text { con } \\
\text { material }\end{array}$} & Prof. & 3.85 & 1.1 & 2.9 & 1.22 & 5.47 & 3.53 & 1.25 & 0.6 \\
\hline & $\begin{array}{l}\text { No } \\
\text { prof. }\end{array}$ & 3.15 & 1.36 & 2.31 & 1.19 & 3.91 & 3.04 & 0.98 & 0.64 \\
\hline \multirow[t]{2}{*}{$\begin{array}{l}\text { Entrena } \\
\text { miento }\end{array}$} & Prof. & 3.79 & 1.1 & 2.96 & 1.17 & 3.96 & 3.24 & 1.26 & 0.69 \\
\hline & $\begin{array}{l}\text { No } \\
\text { prof. }\end{array}$ & 2.88 & 1.4 & 2.12 & 1.31 & 3.13 & 2.79 & 1.05 & 0.71 \\
\hline \multirow[t]{2}{*}{ Material } & Prof. & 3.78 & 1.3 & 3 & 1 & 5.7 & 3.4 & 1.43 & 0.53 \\
\hline & $\begin{array}{l}\text { No } \\
\text { prof. }\end{array}$ & 2.17 & 1.59 & 1.64 & 1.21 & 3.33 & 3.08 & 0.67 & 0.52 \\
\hline \multirow{2}{*}{$\begin{array}{c}\text { Sin } \\
\text { entrena } \\
\text { miento } \\
\text { ni } \\
\text { material }\end{array}$} & Prof. & 2.2 & 1.13 & 2 & 1.41 & 5.8 & 5.02 & 1.14 & 0.69 \\
\hline & $\begin{array}{l}\text { No } \\
\text { prof. }\end{array}$ & 2.4 & 1.47 & 1.64 & 1.33 & 2.11 & 2.65 & 0.63 & 0.5 \\
\hline
\end{tabular}

Finalmente, en la Figura 2, se observan los efectos de interacción entre el promotor de la salud y la condición de entrenamiento sobre las habilidades de las diferentes fases: indaga $(F(7,410)=32.75, p=.000)$, dialoga $(F(7,410)=12.78$, 
$\mathrm{p}=.009)$, establece metas $(F(7,410)=11.17, \mathrm{p}=.008), \mathrm{y}$ acompaña $(F(7,410)=13.25, p=.002)$ en consejo breve.

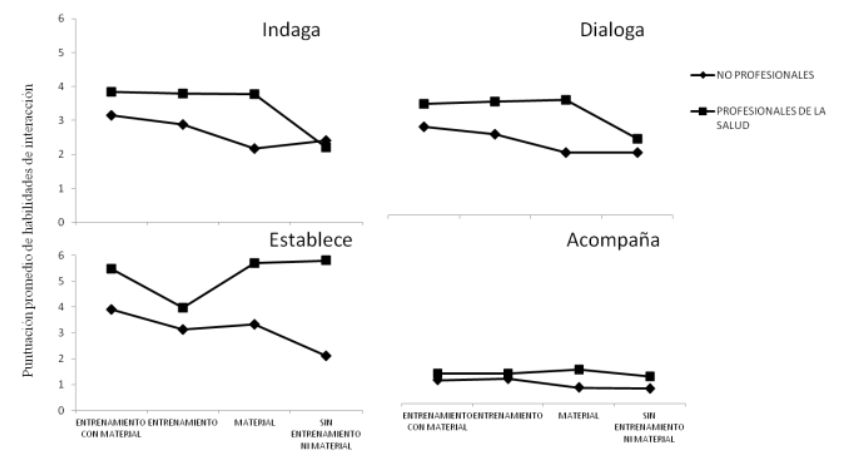

Figura. 2. Efectos de interacción entre el promotor de la salud y la condición de capacitación sobre las habilidades de Indaga (gráfica superior izquierda), dialoga (gráfica superior derecha), Establece metas (gráfica inferior izquierda) y Acompaña (gráfica inferior derecha) en consejo breve.

\section{DISCUSIÓN}

El objetivo de este trabajo fue la evaluación de habilidades de consejo breve de los profesionales y no profesionales de la salud en el programa IDEAS a través de la evaluación de las habilidades prácticas adquiridas mediante situaciones simuladas relacionadas con la interacción promotor usuario y la aplicación de los pasos del programa.

En general, los resultados mostraron que la puntuación promedio fue mayor para los profesionales de la salud en cualquiera de las tres condiciones de entrenamiento. La entrega de material y la participación de los profesionales en el entrenamiento permitió que se adquirieran habilidades de consejo (Whang, Fletcher y Fawcett, 1982), donde se permite, guiar al usuario en la toma de decisiones sin imponer o confrontar al usuario con las consecuencias por su consumo (Hughes y MacNaughton, 2002), sino que en este proceso, el profesional de la salud mostró comportamientos que se asocian con la expresión de sus necesidades y expectativas (Miller y Rollnick, 1999) por parte del usuario, así como con el establecimiento de las metas a alcanzar con el consejo breve (Baer, Wolf y Risley, 1968).

Las puntuaciones promedio elevadas de los profesionales de la salud, reflejaron una mayor adquisición de habilidades de consejo breve que los no profesionales de la salud. Este hallazgo podría ser congruente con la premisa de que los profesionales de la salud muestran los prerrequisitos para la adquisición de habilidades basadas en principios conductuales en la resolución de situaciones hipotéticas en el ámbito de las adicciones, tal como Morales y Vázquez (2011) lo reportaron en el ámbito de la crianza positiva. No obstante, el estudio actual no nos permite afirmar que esta fuera la variable principal que determinara la adquisición de habilidades más alta por los profesionales de la salud. Investigación adicional deberá contemplar el análisis de los conocimientos teóricos, derivados de los principios básicos del comportamiento, previos al entrenamiento en habilidades de consejería breve. Sin embargo, los hallazgos del presente estudio, si permiten conocer la ejecución del comportamiento profesional en comparación con la de los promotores que no pertenecen al campo de la salud; a quienes les fue más complicado adquirir las habilidades de consejo breve con el entrenamiento y los materiales diseñados para ello.

Particularmente, durante las fases Indaga y Dialoga, los resultados mostraron que los profesionales de la salud que no recibieron entrenamiento ni material mostraron una puntación similar a la de los no profesionales. Parece necesario llevar a cabo el entrenamiento y entrega de material, principalmente a promotores con especialización para la ejecución efectiva de las habilidades de consejo breve (Morales y Vázquez, 2011).

El comportamiento "dialogar" en consejo breve, involucra la realización de un balance decisional para incrementar la disposición al cambio de un usuario de drogas a recibir tratamiento (Prochaska y DiClemente, 1984); por lo que tener material para el entrenamiento en esta conducta, siendo profesional de la salud, parece indispensable. Con ello, se obedece a la recomendación de Perepletchikova y Kazdin (2005), quienes señalan la importancia de que el profesional de la salud aprenda y ejecute comportamientos de interacción asociados con la adherencia de los usuarios a los programas de tratamiento y un cambio terapéutico efectivo.

En la etapa "Establece metas" se observó, sin embargo, un efecto importante del papel del participante (profesional de la salud) en la ejecución del consejo breve. En este caso la efectividad de los profesionales de la salud en el establecimiento de metas se aprecia de manera superior al grupo de los no profesionales, y aunque nuevamente se manifiesta la importancia del entrenamiento con material o incluso solo con material, también se observa el efecto en los que no recibieron ninguno de los dos. Es posible que, en esta habilidad, los procedimientos operativos de las instituciones de salud señalen la obligación en la ejecución de esta habilidad del consejo breve (establecimiento de metas) y que aquellos que no son profesionales no se encuentren adheridos a ningún manual de procedimientos que indique la importancia de esta fase. Sin embargo, será indispensable realizar estudios que indiquen qué variables determinaron la eficiencia del establecimiento de metas por los profesionales de la salud, durante el consejo breve.

En la fase de "Acompaña", se pudo observar una diferencia importante en las puntuaciones de los profesionales, no obstante el bajo nivel en ambas muestras para las cuatro condiciones. Aún así, es necesario señalar nuevamente el efecto encontrado en las puntuaciones debido a la disposición 
del material de consejo breve para los profesionales de la salud.

Se puede concluir que para la adquisición de las habilidades prácticas necesarias para la aplicación del programa IDEAS por parte de los promotores, la primera condición es que sean profesionales de la salud, aparentemente con las características de interacción con el usuario (Perepletchikova y Kazdin, 2005) relacionadas con la capacidad de indagar, dialogar, establecer metas con planes de acción y acompañar al usuario (Talavera y Martínez, 2008). Tanto el entrenamiento como la disponibilidad del material de capacitación son necesarios para la adquisición de habilidades, tal como lo muestran los resultados de la mayor parte de los componentes del programa.

El entrenamiento y los materiales, el entrenamiento solo y los materiales utilizados permitieron la adquisición de las habilidades de consejo breve y particularmente las de indagar y dialogar por los profesionales de la salud. El entrenamiento con materiales y los materiales fueron efectivos en la adquisición de las habilidades de establecimiento de metas, para lo cual parece necesario un material o guía de trabajo (aún siendo profesional de la salud). La habilidad de acompañar y averiguar la satisfacción de la persona con el proceso de cambio, parece requerir un proceso de entrenamiento más prolongado con el fin de asegurar el éxito en la adquisición de habilidades, pues las puntuaciones promedio fueron bajas, aun cuando hubo diferencias significativas entre las muestras. Investigación adicional mostrará las estrategias de entrenamiento en el proceso de aprendizaje, necesarias para reducir la latencia de la respuesta de acompañamiento en consejo breve.

Aun cuando los resultados indicaron un aprendizaje mayor en los profesionales de la salud, estudios adicionales podrían mostrar las diferencias adicionales entre los no profesionales de la salud en función de los diversos niveles escolares y su perfil particular. En la actualidad, el consejo breve es otorgado por cualquier persona e incluso por los paraprofesionales y el presente estudio señala la necesidad de desarrollar procedimientos de entrenamiento y específicamente estudios sobre los procesos que faciliten la adquisición de habilidades de consejo breve en esta población.

Finalmente, la investigación adicional en el campo de las adicciones, pero particularmente en el del entrenamiento de los profesionales de la salud, puede generar hallazgos que permitan describir los procesos de aprendizaje que intervienen en términos del perfil y de los conocimientos del consejero en adicciones.

\section{REFERENCIAS}

Baer, D. M., Wolf, M. M. y Risley. T. D. (1968). Some current dimensions of applied behavior analysis. Journal of Applied Behavior Analysis, 1, 91-97.

Bien, T., Miller, W. y Tonigan, J. (1993) Brief interventions for alcohol problems: a review. Addiction, 88, 315-336.

Camacho, S. R. (2012). Cero Alcohol para menores. XI Congreso de Escuelas para Madres y Padres de Familia de Secundarias Técnicas, recuperado el 21 de Noviembre de 2012 en: http://www.eluniversal.com.mx/ciudad/112347.html.

Carrascosa, C. (2007) Terapia Cognitivo Conductual para el tratamiento de las adicciones: fundamentos conceptuales y procedimientos clínicos. México: UNAM.

Cuijpers, P., Riper, H. y Lemmers, L. (2004) The effect on mortality and brief intervention for problem drinkers: a meta-analysis. Addiction, 99(7), 839-845 Doi: 10.1111/J. 1360-0443.

Encuesta Nacional de Adicciones, (2008). Consejo Estatal contra las Adicciones, CONADIC. Recuperado el miércoles 26 de octubre de 2011, de: http://www.conadic.salud.gob.mx/pdfs/ena08/ENA08_NACIONAL.p df.

Hughes P. y MacNaughton G. (2002) Preparing early childhood professionals to work with parents: The challenges of diversity and dissensus. Australian Journal of Early Childhood, 28(2), 14-20.

Instituto Nacional de Psiquiatría Ramón de la Fuente; Instituto Nacional de Salud Pública; Secretaría de Salud. Encuesta Nacional de Adicciones 2011: Reporte de Alcohol. Medina-Mora ME, VillatoroVelázquez JA, Fleiz-Bautista C, Téllez-Rojo MM, Mendoza-Alvarado LR, Romero -Martínez M, Gutiérrez-Reyes JP, Castro-Tinoco M, Hernández-Ávila M, Tena-Tamayo C, Alvear-Sevilla C, y Guisa-Cruz V. México DF, México: INPRFM; 2012. Disponible en www.inprf.gob.mx,www.conadic.gob.mx,www.cenadic.salud.gob.m x,www.insp.mx.

Instituto Nacional de Psiquiatría Ramón de la Fuente; Instituto Nacional de Salud Pública; Secretaría de Salud. Encuesta Nacional de Adicciones 2011: Reporte de Drogas. Villatoro-Velázquez JA, Medina-Mora ME, Fleiz-Bautista C, Téllez-Rojo MM, MendozaAlvarado LR, Romero -Martínez M, Gutiérrez-Reyes JP, CastroTinoco M,, Hernández-Ávila M, Tena-Tamayo C, Alvear-Sevilla C, y Guisa-Cruz V. México DF, México: INPRFM; 2012. Disponible en www.inprf.gob.mx,www.conadic.gob.mx,www.cenadic.salud.gob.m x,www.insp.mx.

Instituto Nacional de Psiquiatría Ramón de la Fuente; Instituto Nacional de Salud Pública; Secretaría de Salud. Encuesta Nacional de Adicciones 2011: Reporte de Tabaco. Reynales-Schigematsu LM, Guerrero-López CM, Lazcano Ponce E, Villatoro-Velázquez JA, Medina-Mora ME, Fleiz-Bautista C, Téllez-Rojo MM, MendozaAlvarado LR, Romero -Martínez M, Gutiérrez-Reyes JP, Castro- 
Tinoco M, Hernández-Ávila M, Tena-Tamayo C, Alvear-Sevilla C, y Guisa-Cruz V. México DF, México: INPRFM; 2012. Disponible en www.inprf.gob.mx,www.conadic.gob.mx,www.cenadic.salud.gob.m x,www.insp.mx.

Liska, H. Miller, P. Nemeth, L. Jenkins, R. Nietert, P. Wessell, A. y Ornstein,

S. (2008) Alcohol screening and brief counseling in a primary care hypertensive population: a cualitative improvement intervention. Addiction 103(8), 1271-1280. Doil 10.1111/J. 13600443.2008.02199.x

Martínez, M. K. (2003). Desarrollo y evaluación de un programa de intervención breve para adolescentes que inician el consumo de alcohol y otras drogas. México: UNAM.

Martínez, K., Pedroza, F., Vacío, M., Jiménez, A. y Salazar, M. (2008). Consejo breve para adolescentes escolares que abusan del alcohol. Revista Mexicana de Análisis de la Conducta, 34(2), 245-262.

Martínez, M.K.I., Salazar, G.M.L., Ruiz, T.G.M., Barrientos, C.V. y Ayala, V.H. (2009). Programa de Intervención Breve para Adolescentes que Inician el Consumo de Alcohol y otras Drogas. Manual del Terapeuta. Consejo Nacional contra las Adicciones, CONADIC. [versión electrónica]. Recuperado el 2 de Noviembre de 2011, de: http://www.conadic.salud.gob.mx/pdfs/publicaciones/ intervencion_breve.pdf.

Medina Mora, M. (2005) Las contribuciones del doctor Ramón de la Fuente en el campo de las adicciones. Salud Mental, 28 (6), 1-8.

Miller, W. R. y Rollnick, S (1999). La Entrevista Motivacional. México: Paidós.

Morales, C. S. y Vazquez, P. F. (2011). Evaluación de conocimientos sobre habilidades de manejo conductual infantil en profesionales de la salud. Acta de Investigación Psicológica, 1(3), 428-440.

Perepletchikova, F. y Kazdin, A. E. (2005). Treatment Integrity and Therapeutic Change: Issues and Research Recommendations. Clinical Psychology: Science and Practice. 12(4), 365-383. doi:10.1093/clipsy/bpi045.

Prochaska, J. O. y DiClemente, C. C. (1984) The Transtheoretical Approach: Towards a Systematic Eclectic Framework. USA: Dow Jones Irwin, Homewood.

Programa Nacional de Salud 2007-2012, recuperado el 25 de Febrero de 2013

http://www.conadic.salud.gob.mx/pdfs/programas/pns version co mpleta.pdf.

Tavera, R. S. y Martínez, R. M. J. (2008). Guía para promotores Nueva vida en Consejo breve: IDEAS. México: CONADIC.

United Nation Office on Drugs and Crime (2010) recuperado el 21 de

noviembre de 2012 en:

http://www.unodc.org/unodc/en/frontpage/2010/April/unodc2010-annual-report-released.html.

Whang, P. L., Fletcher, R. K. y Fawcett, S. B. (1982). Training counseling skills: an experimental analysis and social validation. Journal of Applied Behaviro Analyisis, 15(3), 325-334.
Winters, K. C., Fahnhorst, T., Botzet, A., Lee, S. y Lalone, B. (2012). Brief intervention for drug-abusing adolescents in a school setting: Outcomes and mediating factors. Journal of Substance Abuse Treatment, 42(3), 279-288. Doi: 10.1016/j.jsat.2011.08.005.

\section{APÉNDICE}

\section{LISTA COTEJABLE IDEAS}

Establece un ambiente de confianza y de reserva aclarando que toda la información que proporcione es confidencial.

Informa que la sesión de consejo breve no tiene implicación legal.

Evita hacer juicios de valor con respecto al consumo de sustancias.

Explica las actividades que se realizarán durante la sesión

Identifica el nivel de consumo o dependencia aplicando el cuestionario que corresponde (tabaco/alcohol/drogas)

Indaga en qué situaciones consumo con mayor frecuencia (eventos, personas, días)

Indaga sobre cambios en el comportamiento o sensaciones especiales asociadas al consumo

Indaga sobre las consecuencias negativas asociadas al consumo de la sustancia.

Si el resultado del cuestionario señala dependencia hacia la sustancia canaliza a un centro especializado en el tratamiento de las adicciones

Informa al usuario sobre los resultados obtenidos en el cuestionario

Explica al usuario las consecuencias a corto plazo asociadas al consumo de alcohol y drogas.

Relaciona la información con la posibilidad de desarrollar una adicción

Señala la importancia de elaborar planes de acción que le ayuden a reducir los problemas que ha tenido por consumir sustancias adictivas

Guía al usuario a establecer una meta de consumo

En el caso de tabaco o drogas sugiere la abstinencia, en el caso de alcohol planeta la posibilidad de abstinencia o moderación

Si la meta es moderación explica los límites de consumo recomendados

Si la meta elegida es la moderación explica el concepto de trago estándar

Si la meta elegida es moderación pregunta al usuario qué días de la semana va a consumir

Si la meta elegida es moderación pregunta al usuario cuánto va a consumir

Si la meta elegida es moderación pregunta al usuario en qué condiciones consumirá y cuáles no

Apoya al usuario en el llenado del formato de Establecimiento de Metas junto con el usuario

Pregunta al usuario sobre las cosas que puede hacer para lograr su meta

Apoya al usuario en el análisis de las ventajas y desventajas de cada alternativa

Le sugiere al usuario tips o estrategias para el cumplimiento de su meta Pregunta al usuario sobre la satisfacción hacia la sesión de Consejo Breve

Planea sesiones de seguimiento del proceso para evaluar cambios.

Si no se observan cambios en el consumo del usuario o muestra signos de dependencia canaliza a un centro o institución especializada.

Si observa cambios en el patrón de consumo analiza los planes de acción y las metas establecidas junto con el usuario 